06

\title{
Метод лазерной колорации в экспериментах по филаментации одиночных импульсов и образованию световых пуль в однородных прозрачных диэлектриках
}

\author{
(C) С.В. Чекалин, В.О. Компанец \\ Институт спектроскопии РАН, \\ 108840 Троицк, Москва, Россия \\ e-mail: schekalin@yandex.ru
}

Поступила в редакцию 25.02.2019 г.

В окончательной редакции 25.02.2019 г.

Принята к публикации 15.03.2019 г.

\begin{abstract}
Описаны преимущества метода лазерной колорации для исследования особенностей филаментации в однородных прозрачных диэлектриках и приведены результаты нескольких экспериментов, проведенных этим методом с кристаллом фторида лития. Возможность получать данные по структуре светового поля в филаменте единичного лазерного импульса и обрабатывать их существенно позже проведения эксперимента, практически недоступная другим используемым методам, позволила наблюдать бесплазменный режим филаментации, регистрировать одноцикловые световые пули и показать их робастность.
\end{abstract}

Ключевые слова: световые пули, филаментация, кристаллы LiF, $F$-центры.

DOI: $10.21883 /$ OS.2019.07.47935.64-19

\section{Введение}

Филаментация - явление локализации светового поля при распространении мощного фемтосекундного лазерного излучения в прозрачных диэлектриках, приводящее к сильному росту нелинейно-оптического взаимодействия светового поля со средой $[1,2]$.

Пиковые мощности в фемтосекундных импульсах, генерируемых современными лазерами, могут достигать уровня в несколько петаватт, пиковые интенсивности $10^{13}-10^{14} \mathrm{~W} / \mathrm{cm}^{2}$. При этих условиях нелинейный вклад в показатель преломления среды становится значительным, и эффекты нелинейного самовоздействия, неизбежно сопровождающие распространение высокоинтенсивного излучения в прозрачной среде, приводят к изменениям пространственно-временных и спектральных параметров распространяющихся импульсов. Наиболее существенным это оказывается при филаментации в условиях аномальной дисперсии групповой скорости (ДГС), когда формируются световые пули - экстремально сжатые волновые пакеты с высокой локализацией светового поля не только в пространстве, но и во времени $[1,3]$. С появлением субтераваттных источников сверхкоротких импульсов в перспективном для многих приложений среднем ИК диапазоне спектра, где ДГС многих прозрачных диэлектриков является аномальной, исследование филаментации в изотропных прозрачных диэлектриках становится особенно актуальным для экстремальной лазерной оптики. Важным моментом таких исследований является получение сведений о самых ранних стадиях процесса, определяющих во многих случаях его дальнейшее развитие.
В большинстве работ по филаментации, выполненных до сих пор, такие важные данные как длина филамента или длина пробега световой пули (СП) определялись из фотографий светящихся плазменных каналов, регистрация которых требовала многих лазерных вспышек $[1,4]$. Получаемая при такой методике усредненная картина дает существенные ошибки, особенно на пороге образования филамента. От этого недостатка не свободны и все другие методы, требующие многоимпульсной экспозиции. Метод лазерной колорации (МЛК), описываемый в настоящей работе, дает возможность экспериментального наблюдения динамики одноимпульсной филаментации.

\section{Суть метода МЛК и его преимущества}

Метод основан на известном эффекте окрашивания (колорации) щелочно-галоидных кристаллов при образовании в них люминесцирующих центров окраски (ЦО). Этот эффект первоначально наблюдался при воздействии ионизирующей радиации (рентгеновского излучения или энергичных электронов), а в конце прошлого века и при воздействии лазерного импульса [5], за что и получил название ,лазерная колорация“. По сравнению с другими щелочно-галоидными кристаллами $\mathrm{LiF}$ обладает наиболее интенсивной люминесценцией ЦО, поэтому он используется даже в роли очень чувствительного радиодозиметрического материала. ЦО возникают как при нелинейном фотовозбуждении электронной подсистемы материала с созданием экситонов и электронно-дырочных пар через лавинную, туннельную и многофотонную ионизацию, так и в результате 
прямого возбуждения экситонов [6-8]. Распад экситонов и электронно-дырочных пар приводит к появлению простейших $F$-центров, представляющих собой одиночные анионные вакансии с захваченным электроном [9]. Этот процесс имеет характерное время порядка пикосекунд $[10] . F$-центры в $\mathrm{LiF}$ поглощают свет вблизи $250 \mathrm{~nm}$, но не люминесцируют. После объединения различного числа анионных вакансий и $F$-центров образуются люминесцентные агрегатные ЦО. В LiF возникают долгоживущие центры $F_{2}$ и $F_{3}^{+}$, представляющие собой пару и соответственно тройку анионных вакансий в соседних узлах решетки, захвативших пару электронов. Они имеют почти полностью перекрывающиеся полосы поглощения с максимумами около $450 \mathrm{~nm}$ ( $M$-полоса), а полосы их люминесценции имеют максимумы соответственно около 650 и $550 \mathrm{~nm}$ [11]. Уникальной особенностью этих ЦО в LiF, обнаруженной при воздействии фемтосекундными лазерными импульсами [12], является их столь интенсивная люминесценция, что состоящие из них долгоживущие микроструктуры, созданные при филаментации всего одного лазерного импульса, могут быть легко зарегистрированы и исследованы при последующей подсветке в полосе их поглощения вблизи $450 \mathrm{~nm}$ непрерывным лазером. Это было продемонстрировано в [13], где исследовалась возможность создания люминесцирующих наноэмиттеров при множественной филаментации фемтосекундного единичного лазерного импульса на $800 \mathrm{~nm}$ с пиковой мощностью, на три порядка превышающей критическую мощность самофокусировки для $\mathrm{LiF}$. $\mathrm{LiF}$ имеет высокую термическую и оптическую стабильность ЦО при комнатной температуре, что позволяет исследовать структуры из ЦО различными методами после их записи. Кроме того, $\mathrm{LiF}$ менее гигроскопичен и имеет более удобные для механической обработки свойства по сравнению со многими другими щелочно-галоидными материалами. Еще одно положительное свойство LiF связано с тем, что в отличие от сред с фоточувствительностью, возникающей из-за примесных молекул, доля которых невелика, этот материал обладает собственной естественной фоточувствительностью, что позволяет достичь в эксперименте пространственного разрешения, близкого к атомному. Таким образом, МЛК дает уникальную возможность детального восстановления пространственной картины филаментации после облучения.

Резюмируя, можно перечислить следующие преимущества метода для исследования динамики филаментации и образования СП.

- Во-первых, и это самое существенное, МЛК позволяет подробно исследовать трехмерную структуру оптического поля СП на всей длине ее пробега внутри материала с пространственным разрешением менее $1 \mu \mathrm{m}$ (определяемым исключительно разрешающей способностью используемого микроскопа) после прохождения через материал всего одного импульса. В наиболее эффективном из используемых в настоящее время методов исследования филаментации, так называемом „методе трехмерного изображения“ („three-dimensional imaging technique“ [14]), структура поля регистрируется только на плоскости выходной грани образца с пространственным разрешением не лучше $5 \mu \mathrm{m}$. При этом для получения необходимого количества экспериментальных данных принципиально необходимо накопление сигнала по крайней мере от нескольких сотен лазерных импульсов, что приводит к существенным ошибкам из-за невоспроизводимости параметров лазера от импульса к импульсу.

- Во-вторых, МЛК регистрирует параметры только приосевой части волнового пакета, что важно для исследования непосредственно СП без окружающего ее фона.

- В-третьих, в отличие от всех остальных методов, МЛК не является on-line методом, т.е. структура из долгоживущих центров окраски в $\mathrm{LiF}$, отражающая распределение светового поля в экстремально сжатом волновом пакете, может быть исследована по люминесценции ЦО при подсветке слабым излучением уже намного позже записи этих структур. При этом не регистрируется присутствующее в on-line методах излучение суперконтинуума, конической эмиссии и плазменного канала, что при накоплении сигнала от многих сотен и тысяч лазерных импульсов ведет к неверной оценке длины филамента и длины пробега пули в материале. В МЛК эти величины определяются с использованием только одного лазерного выстрела, и потому однозначно.

- В-четвертых, МЛК чрезвычайно прост как по требуемому оборудованию (кроме возбуждающей лазерной системы, нужен фактически только исследуемый кристалл, микроскоп и светодиод для возбуждения люминесценции), так и по настройке (одна фокусирующая линза), что также сильно увеличивает надежность получаемых результатов по сравнению с системами, использующими много юстируемых зеркал и оптических каналов.

\section{Постановка экспериментов}

Экспериментальные исследования выполнены на спектроскопическом стенде уникальной научной установки УНУ МФЛДСК Института спектроскопии РАН. Схема эксперимента подобна описанной в $[15,16]$. Использовался источник лазерного излучения на основе фемтосекундного генератора Tsunami (Тi:сапфировый лазер) с непрерывным твердотельным лазером накачки Millenia Vs, регенеративного усилителя Spitfire HP с накачкой твердотельным лазером Empower 30 и перестраиваемого параметрического усилителя TOPAS-C с частотой следования импульсов $1 \mathrm{kHz}$. Эксперименты проводились с импульсами на длинах волн 800-3900 nm, перекрывающими области нормальной, нулевой и аномальной ДГC в LiF. Излучение фокусировалось тонкими линзами из $\mathrm{CaF}_{2}$ с фокусным расстоянием от 10 до $30 \mathrm{~cm}$ внутрь образца $\mathrm{LiF}$ на расстоянии нескольких 
миллиметров от его входной грани. Исследования выполнены с импульсами, мощность которых ненамного превышала критическую мощность самофокусировки, что гарантировало режим одного филамента. Длительность импульсов по половине высоты составляла 45, 60, $70 \mathrm{fs}$ соответственно на длинах волн 800, 1250, $1900 \mathrm{~nm}$ и возрастала от 110 до $130 \mathrm{fs}$ на длинах волн 2500-3900 nm. Энергия импульсов измерялась датчиком Fieldmax с детектором PS-10 и для получения одиночной филаментации варьировала от 0.2 до $100 \mu \mathrm{J}$ в зависимости от длины волны.

Эксперименты проводились с образцами LiF размером $3 \times 4 \times 1 \mathrm{~cm} \mathrm{c} \mathrm{полированными} \mathrm{гранями.} \mathrm{Поскольку}$ $\mathrm{LiF}$ имеет кубическую кристаллическую решетку, то в отсутствие механического или электрического напряжений является оптически изотропной средой, что было полностью подтверждено специально проведенными измерениями для всех исследуемых образцов. Появление характерных полос в окрестности 450 и $248 \mathrm{~nm}$ в измеренном нами спектре поглощения структуры, наведенной в $\mathrm{LiF}$, подтверждает возникновение люминесцирующих $F_{2}-, F_{3}^{+}$-центров и нелюминесцирующих $F$-центров $[11,13]$.

Для реализации одноимпульсной экспозиции образец после каждого импульса перемещался в направлении, перпендикулярном лазерному пучку. Для анализа пространственного распределения интенсивности люминесценции записанных ЦО, воспроизводящих плотность лазерно-индуцированных электронных возбуждений в филаменте, использовались методы оптической микроскопии с подсветкой на длине волны поглощения наведенных ЦО. Для качественного анализа записанной структуры из ЦО использовался микроскоп Euromex Oxion 5 с подсветкой непрерывным лазерным излучением на длине волны $450 \mathrm{~nm}$ и регистрацией люминесценции цифровой камерой Nikon D800. Рассеянное возбуждающее излучение отсекалось вспомогательным желто-зеленым светофильтром.

\section{Результаты экспериментов}

На рис. 1 приведены фотографии распределения интенсивности люминесценции ЦО-структур, наведенных одиночным импульсом на длине волны $800 \mathrm{~nm}$ при увеличении энергии импульса от порога появления филамента $(a)$ до возникновения множественной филаментации $(f)$. Фокусное расстояние тонкой $\mathrm{CaF}_{2}$-линзы составляло $280 \mathrm{~mm}$. Отметим, что область высокого поля длиной порядка $100 \mu \mathrm{m}$ и диаметром порядка $10 \mu \mathrm{m}$ (следуя [1], называем ее филаментом, хотя это противоречит определению, данному в [2]) появляется (рис. 1,a) за счет керровского сжатия пучка при интенсивности, еще недостаточной для появления плазмы (мощность импульса порядка $4 P_{\text {cr }}$, где $P_{\text {cr }}=6.58 \mathrm{MW}$ - критическая мощность самофокусировки в $\mathrm{LiF}$ ). Это согласуется с данными работы [17], где сообщается, что при длине волны $830 \mathrm{~nm}$ порог появления суперконтинуума (т.е. возникновения плазмы) выше, чем порог образования ЦО в LiF. Таким образом, МЛК дает уникальную возможность регистрации этого бесплазменного режима самофокусировки, в котором ограничение интенсивности импульса (intensity clamping) происходит не за счет образования плазмы, а вследствие сильного многофотонного поглощения в экситонной полосе $\mathrm{LiF}$ на $12.8 \mathrm{eV}$ [18]. При увеличении мощности импульса до $5 P_{\text {cr }}$ возникает плазма, приводящая к отчетливо наблюдаемому сбоку пространственному расщеплению пучка, принимающего вид „вилки“ (рис. 1, $b-f$ ).

Регистрация структуры пучка методом МЛК с перпендикулярного ракурса показала, что оба тонких филамента, на которые расщепился пучок („зубы“ вилки), лежат в одной плоскости, положение которой не зависит от поляризации лазерного импульса и ориентации образца. Эти филаменты появляются симметрично к оси вначале под углом около $5^{\circ}$ (рис. $\left.1, b\right)$, при росте энергии импульса удлиняются вплоть до $\sim 1 \mathrm{~mm}$, приближаясь к направлению оси, и не испытывают рефокусировок при распространении (рис. $1, e-f)$ вплоть до возникновения множественной филаментации (рис. $1, f$ ). Отсутствие рефокусировок означает отсутствие плазменных каналов, т. е. оба наблюдаемых филамента являются бесплазменными, и поэтому могут быть зарегистрированы только с помощью МЛК. При этом осевая часть пучка с ростом энергии импульса испытывает рефокусировки (рис. $1, e)$. Следует еще раз отметить, что описанная картина зарегистрирована МЛК при воздействии одиночного импульса и вряд ли могла бы наблюдаться при накоплении данных от многих импульсов.

Объяснение наблюдаемого эффекта сводится к следующему. Хорошо известно, что в режиме нормальной ДГС возникающая плазма приводит к расщеплению импульса во времени [1]. При этом задняя часть импульса испытывает положительный фазовый сдвиг изза появления свободных электронов плазмы, с которым, по-видимому, и связано ее пространственное расщепление. Наблюдаемая структура этого расщепления, скорее всего, связана со слабой асимметрией исходного пучка, которая привела к гантелеобразной форме разрушения в плавленом кварце, наблюдавшейся авторами [19] при филаментации мощного одиночного фемтосекундного импульса на длине волны $800 \mathrm{~nm}$. В нашем эксперименте, несмотря на то, что измеряемый пространственный и временной профиль импульса был близок к гауссову, его слабое искажение могло возникнуть изза неидеальной компенсации волнового фронта в паре стретчер-компрессор фемтосекундного усилителя. Сильная нелинейность процесса генерации плазмы приводит к возникновению гораздо большей неоднородности в ней и, как следствие, к асимметрии резервуара энергии, формирующего отклоненные филаменты. В итоге передняя часть расщепленного во времени импульса распространяется по оси, образуя плазменные каналы и испытывая рефокусировки при увеличении энергии. 


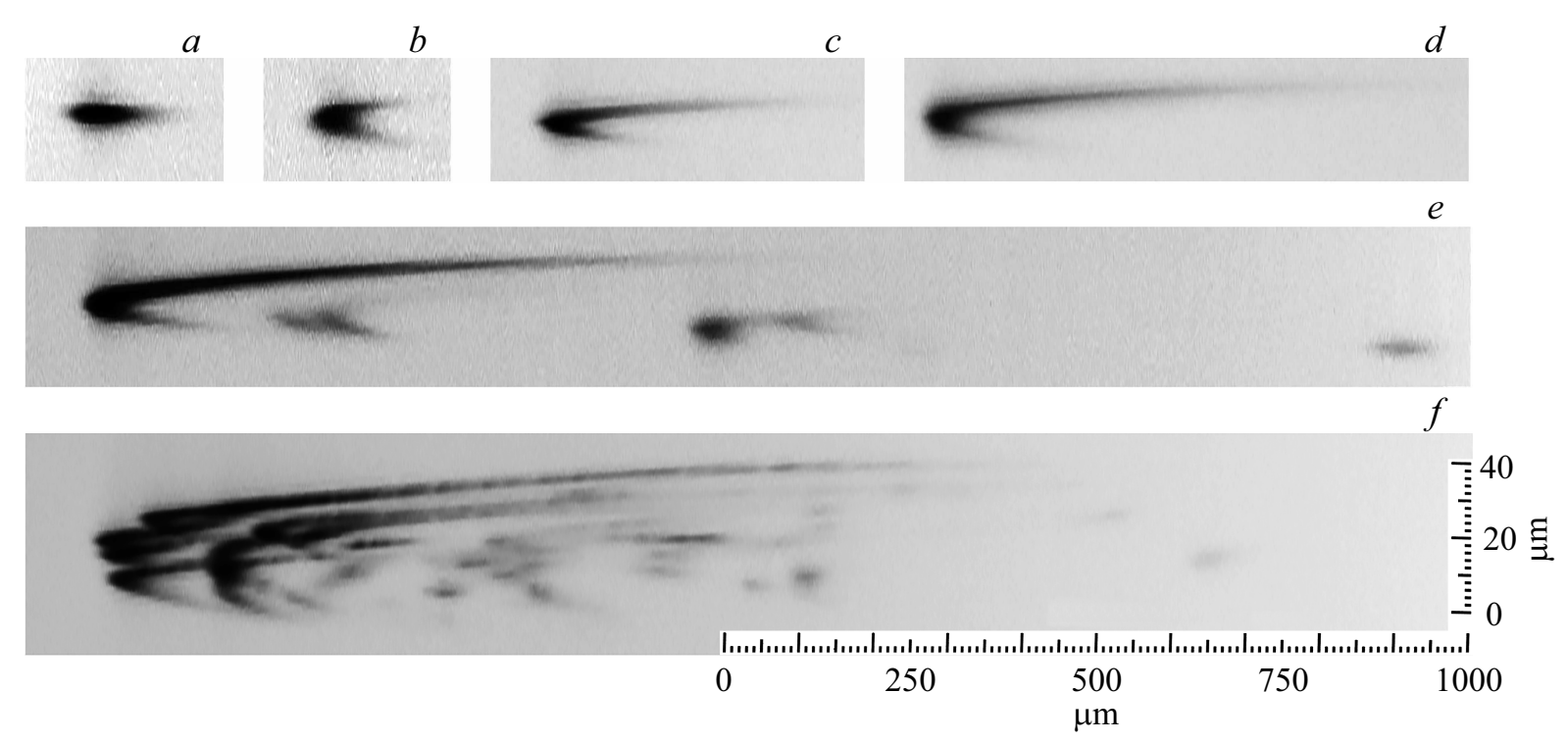

Рис. 1. Фотографии распределения интенсивности люминесценции ЦО-структур, наведенных одиночным $45 \mathrm{fs}$ импульсом на длине волны $800 \mathrm{~nm}$. Импульс распространяется слева направо. Энергия импульса $1.2(a), 1.5(b), 3(c), 5.6(d), 10(e)$ и $30 \mu \mathrm{J}(f)$. Для удобства восприятия вертикальная шкала растянута по сравнению с горизонтальной.

Задний субимпульс расщепляется в пространстве на два тонких „бесплазменных“ филамента, которые распространяются на существенно большие расстояния, чем передний, не испытывая рефокусировок. „Загибание“ их к направлению оси по мере распространения можно объяснить влиянием зоны отрицательного (керровского) сдвига фазы, создаваемой передним импульсом [20].

При энергиях выше $20 \mu \mathrm{J}\left(\sim 150 P_{\text {cr }}\right)$ возникает режим множественной филаментации (рис. $1, f$ ), при котором можно наблюдать появление дополнительных филаментов, направленных от переднего импульса к заднему и демонстрирующих возникновение соответствующих потоков энергии [20].

Еще одним результатом этого эксперимента, обнаруженным с помощью МЛК, является существенно более короткая длина филамента, чем наблюдавшаяся в многоимпульсных измерениях [1-4].

На длинах волн, соответствующих нулевой $(1250 \mathrm{~nm})$ и слабой аномальной $(1900 \mathrm{~nm})$ ДГС, не наблюдалось описанного выше искривления филамента и бесплазменного режима, что может быть связано с увеличением порядка многофотонности и соответствующего возрастания порога появления ЦО за счет прямого возбуждения экситона. Результаты экспериментов по записи структур из ЦО при филаментации импульсов, сфокусированных тонкой линзой с фокусным расстоянием $F=320 \mathrm{~mm}$ для длины волны $1250 \mathrm{~nm}$ и $340 \mathrm{~mm}$ для $1900 \mathrm{~nm}$ в образец $\mathrm{LiF}$ (рис. 2), показали, что характер ДГС существенно влияет на протяженность области высокой плотности энергии светового поля в филаменте. При превышении пиковой мощности над критической мощностью самофокусировки от 2 до 8 раз длина структур из ЦО, записанных в филаменте импульсом на длине волны $1250 \mathrm{~nm}$, возрастает от 78 до $180 \mu \mathrm{m}$, но остается неизменной при записи на длине волны $1900 \mathrm{~nm}$ (рис. 2). В последнем случае эта величина, равная $210 \pm 15 \mu \mathrm{m}$, соответствует длине пробега СП, возникающей в условиях аномальной ДГС. Независимость длины пробега от энергии импульса демонстрирует робастность пули. Следует отметить, что реальная длина пробега СП, зарегистрированная МЛК в одноимпульсном режиме, оказалась на два порядка меньше, чем измерявшаяся в многоимпульсном режиме по длине треков плазменного канала и рассеянного суперконтинуума [21-23].

При переходе в область сильной аномальной ДГС в среднем ИК диапазоне $(2500-3900 \mathrm{~nm})$ МЛК позволил впервые наблюдать образование одноцикловых СП $[15,16]$. Профили плотности ЦО, наведенных при одноимпульсной филаментации в $\mathrm{LiF}$ фемтосекундных импульсов среднего и ближнего ИК диапазонов на длине волны, варьируемой от 800 до $3500 \mathrm{~nm}$, представлены в левой части рис. 3. Видно, что при воздействии одиночного импульса протяженность области с высокой интенсивностью светового поля (т.е. длина филамента) увеличивается с длиной волны, как и на рис. 2. При этом профили филамента остаются довольно гладкими вплоть до длины волны $2600 \mathrm{~nm}$. Начиная с этого значения, дальнейшее увеличение длины волны и соответственно величины аномальной ДГС приводит к появлению в профилях регулярной структуры из ЦО (рис. 3,4 ), возникающей в результате циклической модуляции максимальной амплитуды светового поля пули по мере ее распространения. Модуляция интенсивности люминесценции вдоль оси филамента, соответствующая изменению плотности ЦО с характерным периодом около $30 \mu \mathrm{m}$ (зависящим от длины волны 

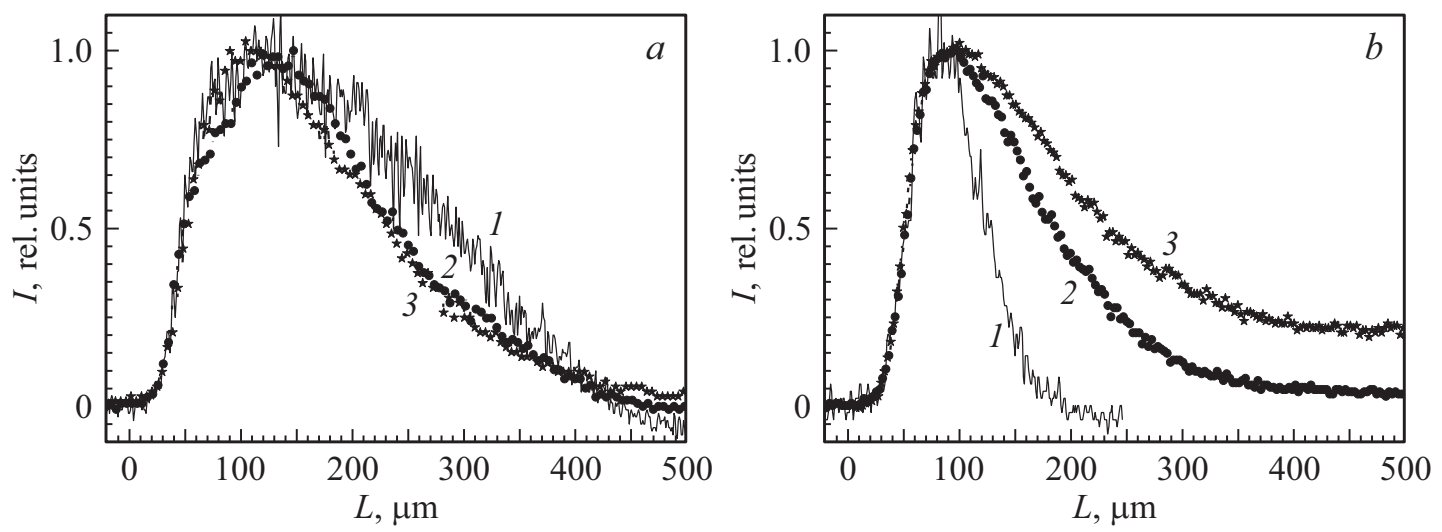

Рис. 2. Профили люминесценции ЦО, наведенных в LiF при одноимпульсной филаментации фемтосекундных импульсов при разных энергиях. Слева: длина волны $1250 \mathrm{~nm}$, энергии 5 (1), 10 (2) и $15 \mu \mathrm{J}$ (3). Справа: длина волны $1900 \mathrm{~nm}$, энергии 7 (1), $14(2)$ и $25 \mu \mathrm{J}(3)$.

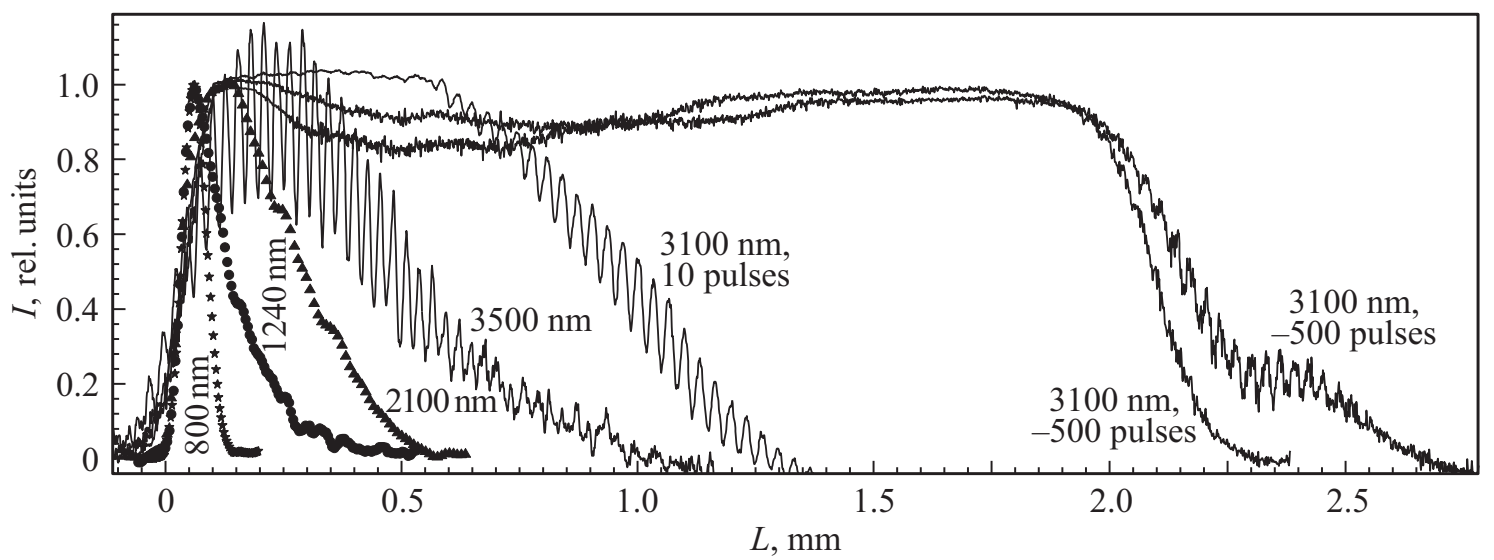

Рис. 3. Профили плотности ЦО, наведенных при филаментации в $\mathrm{LiF}$ фемтосекундных импульсов среднего и ближнего ИК диапазонов на длине волны, варьируемой от 800 до $3500 \mathrm{~nm}$, после прохождения одного импульса, а также 10 и 500 импульсов на длине волны $3100 \mathrm{~nm}$.

ИК импульса $[15,16])$, отражает результат компрессии ИК импульса в СП длительностью, близкой к периоду оптических колебаний [3,22]. Такой процесс характерен только для одноцикловых импульсов и известен с конца прошлого века [24]. Он является результатом периодического изменения сдвига фазы между несущей волной и огибающей волнового пакета вследствие разности групповой и фазовой скоростей. При увеличении числа циклов в волновом пакете эффект модуляции амплитуды светового поля и, как следствие, модуляции плотности наведенных ЦО практически исчезает. Поэтому появление наблюдаемой (рис. 3,4) регулярной модуляции профиля плотности ЦО, наведенных при филаментации в LiF, свидетельствует о формировании одноцикловой СП. Что касается длины ее пробега, то она, судя по данным эксперимента растет с длиной волны, но не превышает нескольких сотен микрон, что соответствует времени жизни в единицы пикосекунд. Важно отметить, что вид трека СП не меняется при увеличении энергии лазерного импульса от порога появления филамента до возникновения второй СП, структура которой в точности совпадает со структурой первой СП, что свидетельствует о ее робастности.

Возникновение долгоживущих ЦО в LiF сопровождается увеличением показателя преломления на оси филамента и образованием оптического волновода в объеме диэлектрика [13]. Использование многоимпульсного возбуждения в МЛК позволяет реализовать волноводный режим распространения СП, практически не исследованный в однородных материалах. На рис. 3 представлены результаты измерения ЦО-структур, полученных как в одноимпульсном, так и в многоимпульсном режимах. В последнем случае долгоживущие волноводы создавались различным числом импульсов с низкой частотой следования $(4 \mathrm{~Hz})$ для исключения тепловых эффектов, а затем без изменения положения образца регистрировалась картина возникновения и распространения СП для различных размеров наведенных волноводов. На рис. 3 можно видеть, что одноцикловая СП появляется на выходе волновода длиной до нескольких миллиметров, 


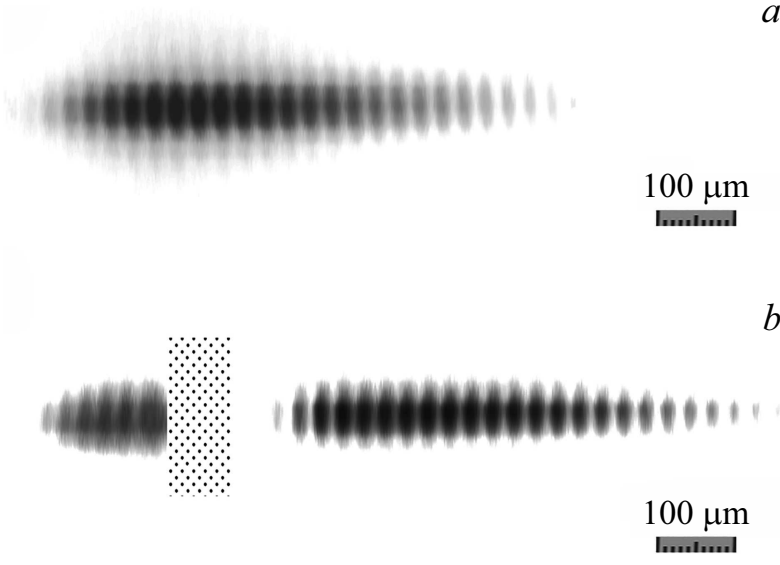

Pис. 4. Фотографии распределения интенсивности люминесценции ЦО-структур, наведенных одноцикловой СП (трек пробега пули), при филаментации одиночного $120 \mathrm{fs}$ импульса на длине волны $3200 \mathrm{~nm}$. Энергия импульса $25 \mu \mathrm{J}$. Импульс распространяется слева направо. Вид трека пробега $(a)$ в однородной среде; $(b)$ в среде с воздушным зазором (заштрихованная область). Для лучшего восприятия вертикальный размер растянут в 10 раз.

наведенного десятками и даже сотнями импульсов. Однако с ростом числа импульсов вероятность этого события падает - после 500 импульсов СП появляется не всегда. Тем не менее можно утверждать, что одноцикловая СП не меняет своих параметров после прохождения наведенного волновода. Такой же эффект наблюдался при прохождении воздушного промежутка (рис. 4) и полном внутреннем отражении одноцикловой СП в наших экспериментах, проведенных с помощью МЛП. Это еще раз подтверждает устойчивость СП, являющуюся следствием самоорганизации интенсивного светового поля при его нелинейно-оптическом взаимодействии со средой в условиях аномальной ДГС $[15,16]$.

Важный, но еще недостаточно изученный аспект филаментации связан с фазой огибающей несущей частоты при распространении близких к одноцикловым импульсов в прозрачной среде [25,26]. Регулярное изменение параметров близких к одноцикловым СП среднего ИК диапазона при распространении в кристалле LiF, наблюдаемое с помощью МЛК, позволяет проследить за непрерывно изменяющимся сдвигом фазы огибающей несущей частоты, измерение которого является одной из наиболее актуальных проблем современной аттосекундной оптики [27].

\section{Финансирование работы}

Исследование выполнено за счет гранта Российского научного фонда (проект № 18-12-00422).

\section{Конфликт интересов}

Авторы заявляют, что у них нет конфликта интересов.

\section{Список литературы}

[1] Couairon A., Mysyrowicz A. // Phys. Rep. 2007. V. 441. P. 47. doi 10.1016/j.physrep.2006.12.005

[2] Chin S.L. Femtosecond Laser Filamentation. Springer Series on Atomic, Optical and Plasma Physics. Springer, 2010. V. 55.

[3] Чекалин С.В., Компанеи, В.О., Дормидонов А.Е., Кандидов В.П. // УФН. 2019. Т. 189. № 3. doi $10.3367 /$ UFNr.2018.06.038419

[4] Чекалин С.В., Компанеи, В.О., Докукина А.Э., Дормидонов А.Е., Сметанина Е.О., Кандидов В.П. // Квант. электрон. 2015. T. 45. № 5. C. 401; Chekalin S.V., Kompanets V.O., Dokukina A.E., Dormidonov A.E., Smetanina E.O., Kandidov V.P. // Quantum Electron. 2015. V. 45. N 5. P. 401. doi 10.1070/QE2015v045n05ABEH015773

[5] Bichevin V. Kaambre H. // Phys. Status Solidi B. 1994. V. 186. P. 57. doi 10.1002/pssb.2221860104

[6] Stuart B.C., Feit M.D., Rubenchik A.M., Shore B.W., Perry M.D. // J. Opt. Soc. Am. B. 1996. V. 13. P. 459. doi 10.1364/JOSAB.13.000459

[7] Kaiser A., Rethfeld B., Vicanek M., Simon G. // Phys. Rev. B. 2000. V. 61. P. 11437. doi 10.1103/PhysRevB.61.11437

[8] Mao S.S., Quéré F., Guizard S., Mao X., Russo R.E., Petite G., Martin P. // Appl. Phys. A. 2004. V. 79. P. 1695. doi 10.1007/s00339-004-2684-0

[9] Лущик Ч.Б., Лущик А.Ч. Распад электронных возбуждений с образованием дефектов в твердых телах. М.: Наука, 1989.

[10] Hirai M., Suzuki Y., Okumura M. // J. Phys. Colloques. 1980. V. 41. P. C6. doi 10.1051/jphyscol:1980677

[11] Baldacchini G. // J. Luminesc. 2002. V. 100. P. 333. doi 10.1016/S0022-2313(02)00460-X

[12] Kurobori Toshio, Kawamura Ken-ichi, Hirano Masahiro, Hosono Hideo. // J. Phys.: Condens. Matter. 2003. V. 15. P. L399. doi 10.1088/0953-8984/15/25/101

[13] Мартынович Е.Ф., Кузнецов А.В., Кирпичников А.В., Пестряков Е.В., Багаев С.Н. // Квант. электрон. 2013. T. 43. № 5. C. 463; Martunovich E.F., Kuznetsov A.V., Kirpichnikov A.V., Pestryakov E.V., Bagayev S.N. // Quantum Electron. 2013. V. 43. N 5. P. 463. doi 10.1070/QE2013v043n05ABEH015117

[14] Majus D., Tamošauskas G., Gražulevičiūtè I., Garejev N., Lotti A., Couairon A., Faccio D., Dubietis A. // Phys. Rev. Lett. 2014. V. 112. P. 193901. doi 10.1103/PhysRevLett.112.193901

[15] Кузнецов А.В., Компанеи В.О., Дормидонов А.Е., Чекалин С.В., Шленов С.А., Кандидов В.П. // Квант. электрон. 2016. V. 46. № 4. P. 379; Kuznetsov A.V., Kompanets V.O., Dormidonov A.E. et al. // Quantum Electron. 2016. V. 46. N 4. P. 379. doi 10.1070/QEL16038

[16] Chekalin S.V., Kompanets V.O., Kuznetsov A.V., Dormidonov A.E., Kandidov V.P. // Laser Phys. Lett. 2016. V. 13. P. 065401. doi 10.1088/1612-2011/13/6/065401

[17] Courrol L.C., Samad R.E., Gomes L., Ranieri I.M., Baldochi S.L., de Freitas A.Z., Vieira N.D. Jr. // Optics Express. 2004. V. 12. N 2. P. 288. doi 10.1364/OPEX.12.000288

[18] Rohlfing M., Louie S.G. // Phys. Rev. Lett. 1998. V. 81. N. 11. P. 2312. doi 10.1103/PhysRevLett.81.2312

[19] Yashunin D.A., Malkov Yu.A., Mochalov L.A., Stepanov A.N. // J. Appl. Phys. 2015. V. 118. P. 093106. doi 10.1063/1.4929649

[20] Šiaulys N., Melninkaitis A., Dubietis A. // Opt. Lett. 2015. V. 40. N 10. P. 2285. doi 10.1364/OL.40.002285 
[21] Чекалин С.В., Докукина А.Э., Сметанина Е.О., Компанеи, В.О., Кандидов В.П. // Квант. электрон. 2014. Т. 44. № 6. C. 570; Chekalin S.V., Dokukina A.E., Smetanina E.O., Kompanets V.O., Kandidov V.P. // Quantum Electron. 2014. V. 44. N 6. P. 570. doi 10.1070/QE2014v044n06ABEH015452

[22] Чекалин С.В., Компанеи В.О., Дормидонов А.Е., Кандидов В.П. // Квант. электрон. 2018. Т. 48. № 4. C. 372; Chekalin S.V., Kompanets V.O., Dormidonov A.E., Kandidov V.P. // Quantum Electron. 2018. V. 48. N 4. P. 372. doi 10.1070/QEL16644

[23] Dokukina A.E., Smetanina E.O., Kompanets V.O., Chekalin S.V., Kandidov V.P. // Proc. SPIE. 2014. V. 9219. P. 92190I.

[24] Xu L., Spielmann C., Poppe A., Brabec T., Krausz F., Hänsch T.W. // Opt. Lett. 1996. V. 21. P. 2008.

[25] Gong C., Jiang J., Li C., Song L., Zeng Z., Zheng Y., Miao J., Ge X., Deng Y., Li R., Xu Z. // Opt. Exp. 2013. V. 21. P. 24120.

[26] Grazuleviciute I., Suminas R., Tamosauskas G., Couairon A., Dubietis A. // Opt. Lett. 2015. V. 40. P. 3719.

[27] Krausz F., Ivanov M. // Rev. Mod. Phys. 2009. V. 81. P. 163. 